Muro de la Investigación, 2021(2), julio-diciembre ISSN: 2523-2886

DOI:https://doi.org/ 10.17162/rmi.v6i2.1639

\title{
Apoyo social y dependencia funcional en adultos mayores de seis iglesias adventistas del séptimo Día
}

\author{
Social support and functional dependency in older adults from six \\ Seventh-day Adventist churches
}

\author{
Yuzzelli Lourdes Castilla Anglas ${ }^{1 a}$ y Lucero Pardavé Machoa ${ }^{2}$ \\ Universidad Peruana Unión, Lima, Perú ${ }^{12}$
}

Recibido: 12 de noviembre de 2020

Aceptado: 19 de junio de 2021

\begin{abstract}
Resumen
El objetivo de este artículo ${ }^{\mathrm{a}}$ es determinar la relación entre apoyo social y dependencia funcional en adultos mayores. El estudio es de enfoque cuantitativo, diseño no experimental, tipo correlacional y de corte transversal. La muestra de selección se realizó mediante el muestreo no probabilístico por conveniencia, la cual estuvo conformada por 150 adultos mayores. Se utilizó dos instrumentos para la recolección de datos, el cuestionario de apoyo social de MOS adaptado por Cruz (2009) con una prueba de confiabilidad de 0,97 y su vez la escala Lawton y Brady, adaptado por Meza (2016). Se encontró que el 38,7\% presentan un nivel bajo de apoyo social y un 30,7\% tiene entre un nivel medio y alto; por otro lado, en el análisis de cada dimensión, presentaron un nivel bajo en apoyo emocional (43,3\%), apoyo instrumental $(46,7 \%)$, en interacción social $(53,3 \%)$ entre alto y medio, apoyo afectivo $(38,8 \%)$ alto. El $46,7 \%$ con dependencia moderada, un $44 \%$ es funcionalmente independientes, el $4,7 \%$ entre dependencia total y dependencia severa. En conclusión, existe relación significativa entre ambas variables.
\end{abstract}

Palabras Clave: apoyo social, dependencia funcional, adulto mayor, envejecimiento, geriatría, enfermería.

\begin{abstract}
The objective of this article is to determine the relationship between social support and functional dependence in older adults. The study has a quantitative approach, non-experimental design, correlational and cross-sectional type. The selection sample was made through non-probabilistic sampling for convenience, it was made up of 150 older adults. Two instruments were used for data collection, the MOS social support questionnaire adapted by Cruz (2009) with a reliability test of 0.97 and the Lawton and Brady scale, adapted by Meza (2016). It was found that $38.7 \%$ have a low level of social support and $30.7 \%$ have a medium and high level, on the other hand, in the analysis of each dimension, they presented a low level in emotional support (43.3\%), instrumental support $(46.7 \%)$, in social interaction $(53.3 \%)$ between high and medium, emotional support $(38.8 \%)$ high. $46.7 \%$ with moderate dependence, $44 \%$ are functionally independent, $4.7 \%$ between total
\end{abstract}

\footnotetext{
${ }^{a}$ Email: yuzzellicastilla@upeu.edu.pe. La presente investigación se basa en la tesis de maestría de las autoras: http://hdl.handle.net/20.500.12840/3147
} 
dependence and severe dependence. In conclusion: there is a significant relationship between both variables.

Keywords: social support, functional dependence, elderly, aging, geriatrics, nursing.

\section{Introducción}

Según la Organización Mundial de la Salud (OMS, 2020), entre los años 2000 y 2050, la cantidad de la población mundial que tiene 60 años de edad o más se duplicará, y pasará de un 11 al $22 \%$. Asimismo, se prevé que este número absoluto de personas aumentará de 900 millones, en el 2015 a 1400 millones para el 2030, y 2100 millones para el 2050; que podrían llegar a los 3200 millones en el 2100.

Asimismo, la Organización Mundial de la Salud (OMS, 2020), con el incremento de esta población, se pronostica que de aquí al año 2050 la cantidad de ancianos que no puedan valerse por sí mismos se multiplicará por cuatro en los países en desarrollo. Es así que, muchos ancianos de edad muy avanzada perderán la capacidad de vivir independientemente a causa de padecer limitaciones de la movilidad, fragilidad u otros problemas físicos o mentales. Así también, muchos necesitarán alguna forma de asistencia a largo plazo, ya sean cuidados domiciliarios o comunitarios, reclusión en asilos y estadías prolongadas en hospitales.

Según la Organización Panamericana de la Salud, en América Latina y el Caribe, se calcula que la cantidad de adultos mayores se duplicará en el 2030, representando un 16,7\% de la población (OPS \& OMS, 2015). Por otro lado, en el Perú, existe un aumento de la población adulta mayor de $5,7 \%$, en el año 1950 a 10,1\%, en el 2017. De igual manera, se considera que en los hogares de Lima Metropolitana el 37,7\% cuenta con un adulto mayor, según el Instituto Nacional de Estadística e Informática (INEI, 2017).

Por otro lado, en el análisis situacional del Perú (ASIS, 2012), los problemas de morbilidad que aquejan a este grupo etario son: las enfermedades crónicas degenerativas, las infecciones respiratorias agudas bajas, los accidentes cerebrovasculares, la hipertensión arterial y las patologías isquémicas cardiacas. Del mismo modo, las muertes por diabetes mellitus representaron el 3,9\%, seguidas de la insuficiencia renal, la cirrosis y otras enfermedades crónicas hepáticas (Ministerio de Salud y Dirección General de Epidemiología, 2013).

Seguidamente, Villalobos (2014) considera al adulto mayor como una población vulnerable a cualquier alteración familiar, social, económica o de otro tipo, lo que compromete rápidamente su estado de salud, evidenciándose en la limitación de su capacidad funcional o dependencia física, además de su autoestima. La pérdida de roles en los adultos mayores ha implicado también, pérdidas 
de espacio y de pertenencia y esto ha contribuido a generar algún grado de indefensión del adulto mayor que se expresa muchas veces en desamparo y abandono, llegando a ser una carga para la familia y la sociedad.

Por su parte, el Instituto Nacional de Estadística e Informática (INEI, 2018) reporta que, en el Perú, existen 633 mil 590 adultos mayores que viven solos, representando el 38,4\%. Cabe precisar, que, de este total, un 61,8\% vive con otra persona igualmente anciana, que puede ser el cónyuge, algún familiar o amigo. A su vez, un 38,2\% viven completamente solos, es decir, en un hogar unipersonal. Además, conforme avanzan los años, el adulto mayor se va deteriorando y su estado funcional no es el mismo ocasionando que las actividades que antes realizaba a un ritmo normal, se realicen de distinto modo, debido a que la pérdida de masa y función muscular ocasiona disminución en la fuerza, siendo predictor incluso de morbilidad y mortalidad (Meza, 2016).

En función a los principios cristianos de la Iglesia Adventista del Séptimo Día (IASD) que señalan los mandamientos "Honra a tu padre y a tu madre" y el "amor al prójimo", la iglesia practica el cuidado y respeto hacia el adulto mayor incluyéndolos en actividades de la iglesia como: ancianos, diáconos, el ministerio de la mujer, la escuela sabática, etc. que los mantienen desarrollándose en varias actividades, evidenciando buena capacidad funcional a pesar de la edad. Por lo cual nos planteamos la siguiente pregunta de investigación ¿Cuál es la relación de apoyo social que reciben los adultos mayores según el grado de dependencia funcional?

\title{
Metodología
}

\section{Diseño y tipo de investigación}

La presente investigación es de enfoque cuantitativo porque mide variables numéricas, empleando la estadística para probar hipótesis de estudio. El diseño es no experimental, porque no se manipulan deliberadamente las variables. De tipo correlacional, ya que busca la relación entre la variable apoyo social y dependencia funcional. Por último, de corte transversal porque la medición se

llevó a cabo en un solo momento (Hernández, Fernández \& Baptista, 2014). Gráficamente el diseño se representa de la siguiente manera:

\author{
Donde: \\ $\mathrm{X}=$ apoyo social \\ $\mathrm{Y}=$ dependencia funcional $\mathrm{r}=$ relación
}




\section{Delimitación geográfica}

La urbanización El Inti pertenece al distrito de Lurigancho-Chosica y alberga a 6 iglesias adventistas del séptimo día, haciendo un total de aproximadamente 1200 feligreses, de los cuales 200 son adultos mayores de 60 años.

\section{Participantes}

La población estuvo conformada por 200 adultos mayores de 60 años de seis iglesias adventistas: El Inti, Alameda III, Jerusalén, Villa La Era, Colinas y Redención. La muestra estuvo conformada por 150 adultos mayores, muestreo no probabilística y por conveniencia, ya que no se siguieron los supuestos de aleatoriedad, y fue de carácter censal, porque toda la población fue considerada como muestra (Hernández, Fernández \& Baptista, 2014).

\section{Criterios de inclusión}

Adultos mayores de 60 años que sean participantes de las iglesias: El Inti, Alameda III, Jerusalén, Villa La Era, Colinas y Redención.

\section{Criterios de exclusión}

Que sean adultos menores de 60 años, que no firmen el consentimiento informado.

\section{Características de la muestra}

En la tabla 1, se observa que del 100\% de la población encuestada, el 54\% fueron de género femenino, el 66\% tuvieron entre 60 y 71 años, el 58,7\% manifestaron estar casados y el 72\% tuvieron de 3 a 4 hijos. De igual manera, el 44,7\% contaron con estudios de primaria, el 64,7\% viven con su esposo y/o hijos, el 74,3\% no trabaja y el 43,3\% cuenta con seguro SIS y el $88 \%$ practica la religión adventista. 


\section{Tabla 1}

Características sociodemográficas

\begin{tabular}{|c|c|c|c|}
\hline Variable & Escala & $\mathrm{n}$ & $\%$ \\
\hline \multirow[t]{2}{*}{ Genero } & Femenino & 81 & 54,0 \\
\hline & Masculino & 69 & 46,0 \\
\hline \multirow[t]{3}{*}{ Edad } & De 60 a 71 años & 99 & 66,0 \\
\hline & De 72 a 83 años & 39 & 26,0 \\
\hline & De 84 a más & 12 & 8,0 \\
\hline \multirow[t]{3}{*}{ Hijos } & Ningún hijo & 14 & 9,3 \\
\hline & De 1 a 2 hijos & 28 & 18,7 \\
\hline & De 3 a 4 hijos & 108 & 72,0 \\
\hline \multirow[t]{4}{*}{ Estado civil } & Casado/a & 88 & 58,7 \\
\hline & Divorciado/a & 12 & 8,0 \\
\hline & Soltero/a & 26 & 17,3 \\
\hline & Viudo/a & 24 & 16,0 \\
\hline \multirow[t]{4}{*}{ Lugar de procedencia } & Costa & 53 & 35,3 \\
\hline & Sierra & 89 & 59,3 \\
\hline & Selva & 4 & 2,7 \\
\hline & Extranjero & 4 & 2,7 \\
\hline \multirow[t]{4}{*}{ Grado de instrucción } & Primaria & 67 & 44,7 \\
\hline & Secundaria & 56 & 37,3 \\
\hline & Técnico & 14 & 9,3 \\
\hline & Universitario & 13 & 8,7 \\
\hline \multirow[t]{4}{*}{ Con quien vive } & Solo & 21 & 14,0 \\
\hline & Esposo y/o hijos & 97 & 64,7 \\
\hline & Familiares & 26 & 17,3 \\
\hline & Amigos & 6 & 4,0 \\
\hline \multirow{2}{*}{ Ocupación } & Trabaja & 39 & 26,0 \\
\hline & No trabaja & 111 & 74,0 \\
\hline \multirow[t]{3}{*}{ Seguro de salud } & SIS & 65 & 43,3 \\
\hline & EsSalud & 62 & 41,3 \\
\hline & Otro & 23 & 15,3 \\
\hline \multirow[t]{2}{*}{ Religión } & $\mathrm{Si}$ & 132 & 88,0 \\
\hline & No & 18 & 12,0 \\
\hline Total & & 150 & 100,0 \\
\hline
\end{tabular}

\section{Técnica e instrumento de recolección de datos}

La técnica de recolección de datos, para medir la variable apoyo social, fue la encuesta y para la dependencia funcional la entrevista. El cuestionario de apoyo social de MOS fue diseñado por Sherbourne y Stewart (1991) en los Estados Unidos como un cuestionario breve y autoadministrado 
en personas con enfermedades crónicas, a partir de los datos del estudio del grupo The Medical Outcomes Study Social Support Survey (MOS-SSS), traducido al español por Revilla et. al (2005), en Argentina, en pacientes con enfermedades crónicas. Del mismo modo, fue adaptado en el Perú por Cruz (2009), con un Alpha de Cronbach de 0,97.

El instrumento consta de 20 ítems distribuidos en 4 dimensiones: apoyo emocional (2,3,7,8,12,15,16,18 ítems), apoyo instrumental (1,4,11,14 ítems), apoyo social positivo $(6,10,13,17$ ítems) y apoyo afectivo (5,9,19 ítems). Las alternativas de respuesta van en escala tipo Likert de 1 a 5 puntos (nunca, casi nunca, a veces, casi siempre y siempre); y la escala de puntuación final lo clasifica en: alto (95-75 pts), medio (74-52 pts) y bajo (51-19 pts). Villalobos (2014) realizó la confiabilidad del instrumento en un estudio realizado en Perú, contando con un Alpha de Cronbach de 0,7.

\section{Escala de Lawton y Brody}

Para la variable dependencia funcional, se utilizó Escala de Lawton y Brody (1969) que se desarrolló en Philadelfia Geriatric Center para la evaluación de autonomía física AIVD en población anciana institucionalizada o no. Traducido y adaptado en España (1993) y adaptada en el Perú por Meza (2016) con valor de Alpha de Cronbach de 0,8. La escala Lawton y Brody valora la capacidad de la persona para realizar las actividades instrumentales (actividades necesarias para vivir de manera independiente en la comunidad como hacer la compra, preparar la comida, manejar dinero, usar el teléfono, tomar medicamentos, entre otros). El proceso de recolección será a través de un cuestionario aplicado por el investigador, el cual consta de 8 ítems y discrimina los ítems 3,4 y 5 si son varones. La escala de calificación final lo clasifica en: Dependencia total (0-1 pts), grave (2-3 pts), moderada (4-5 pts), leve (6-7 pts) y autonomía (8 pts).

\section{Proceso de recolección de datos}

Se solicitó a la E.P Enfermería una carta de presentación dirigida a la directiva de las iglesias: El Inti, Alameda III, Jerusalén, Villa La Era, Colinas y Redención). Seguidamente, se presentó el proyecto en dichas entidades y se solicitaron los permisos necesarios. Una vez logrado esto, se procedió a coordinar con el Pr. Carlos Chumbes y los ancianos de iglesia las fechas para la aplicación de los instrumentos. La información fue levantada el mes de enero de 2020, aplicando los instrumentos previos a la firma de consentimiento informado y tomando un tiempo de 20 min por persona.

\section{Procesamiento y análisis de datos}

Los datos fueron tratados haciendo uso del software Statistics y el paquete estadístico SPSS versión 23; el ingreso de la misma, se realizó después de la elaboración de la plantilla en el software, 
considerando la codificación original de los instrumentos y previo foliado para evitar errores de usuario al momento de ingresar los datos. Se realizó la limpieza de datos verificando la fiabilidad del instrumento. Posteriormente, se cumplió con el procesamiento de la prueba Kolmogorov-Smirnov para ver si los datos se ajustaban a una distribución normal (ver anexo 4), al ver que los datos no alcanzaron dicho supuesto, se empleó la prueba Rho de Spearman para hallar la relación entre las variables, utilizando el valor numérico de ambas.

\section{Resultados}

Tabla 2

Relación entre apoyo social y dependencia funcional en adultos mayores de seis iglesias adventistas del séptimo día, Lurigancho Chosica, 2019

\begin{tabular}{|c|c|c|c|c|c|c|c|}
\hline & & & \multirow[b]{2}{*}{ DT } & \multicolumn{2}{|c|}{ Dependencia funcional } & \multirow[b]{2}{*}{ I } & \multirow[b]{2}{*}{ Total } \\
\hline & & & & DS & $\mathrm{DM}$ & & \\
\hline Aроуо & Bajo & $\mathrm{n}$ & 7 & 4 & 30 & 17 & 58 \\
\hline \multirow[t]{5}{*}{ Social } & & $\%$ & $4,7 \%$ & $2,7 \%$ & $20,0 \%$ & $11,3 \%$ & $38,7 \%$ \\
\hline & Medio & $\mathrm{n}$ & 0 & 2 & 18 & 26 & 46 \\
\hline & & $\%$ & $0,0 \%$ & $1,3 \%$ & $12,0 \%$ & $17,3 \%$ & $30,7 \%$ \\
\hline & Alto & $\mathrm{n}$ & 0 & 1 & 22 & 23 & 46 \\
\hline & & $\%$ & $0,0 \%$ & $0,7 \%$ & $14,7 \%$ & $15,3 \%$ & $30,7 \%$ \\
\hline \multirow[t]{2}{*}{ Total } & & $\mathrm{n}$ & 7 & 7 & 70 & 66 & 150 \\
\hline & & $\%$ & $4,7 \%$ & $4,7 \%$ & $46,7 \%$ & $44,0 \%$ & $100,0 \%$ \\
\hline
\end{tabular}

Rho $=.301 ; \mathrm{p}=.000$

En la tabla 2, se observa que del 100\% de la población, el 17,3\% de adultos mayores tiene un apoyo social de nivel medio y son funcionalmente independientes; por otro lado, el $0,7 \%$ de adultos mayores tiene un nivel alto de apoyo social y son funcionalmente dependientes. Con respecto a la relación entre variables, se observa que el estadístico $r$ de Pearson evidencia un coeficiente de correlación de 0,301 y un p valor de 0,000, siendo este menor a 0,05, lo que permite aceptar la H1 y rechazar H0, quedando demostrando que existe relación significativa entre el apoyo social y la dependencia funcional en adultos mayores de seis iglesias adventistas del séptimo día. 
Tabla 3

Apoyo social en adultos mayores de seis iglesias adventistas del séptimo día,

Lurigancho-Chosica, 2019

\begin{tabular}{lll}
\hline Apoyo social & $\mathrm{n}$ & $\%$ \\
\hline Bajo & 58 & 38,7 \\
Medio & 46 & 30,7 \\
Alto & 46 & 30,7 \\
Total & 150 & 100,0 \\
\hline
\end{tabular}

En la tabla 3, se observa que del 100\% de los adultos mayores encuestados, el 38,7\% presentan un nivel bajo de apoyo social y un 30,7\% tiene entre un nivel medio y alto de apoyo social.

\section{Tabla 4}

Dependencia funcional en adultos mayores de seis iglesias adventistas del séptimo día, Lurigancho-Chosica, 2019

\begin{tabular}{lll}
\hline Dependencia funcional & $\mathrm{n}$ & $\%$ \\
\hline Dependencia total & 7 & 4,7 \\
Dependencia severa & 7 & 4,7 \\
Dependencia moderada & 70 & 46,7 \\
Independencia & 66 & 44,0 \\
Total & 150 & 100,0 \\
\hline
\end{tabular}

En la tabla 4, se puede observar que del $100 \%$ de los adultos mayores encuestados, el 46,7\% presenta dependencia moderada, mientras que el $44 \%$ es independiente; por otra parte, el $4,7 \%$ se encuentra entre la dependencia total y dependencia severa. 


\section{Tabla 5}

Relación entre la dimensión apoyo emocional y dependencia funcional en adultos mayores de seis iglesias adventistas del séptimo día, Lurigancho Chosica, 2019

\begin{tabular}{ccccc}
\hline & \multicolumn{5}{c}{ Dependencia funcional } \\
\cline { 2 - 5 } & DT & DS & DM & I \\
\hline 7 & 5 & 32 &
\end{tabular}

\begin{tabular}{|c|c|c|c|c|c|c|}
\hline Apoyo Bajo & $\mathrm{n}$ & & & & 21 & 65 \\
\hline Emocional & $\%$ & $4,7 \%$ & $3,3 \%$ & $21,3 \%$ & $14,0 \%$ & $43,3 \%$ \\
\hline Medio & $\mathrm{n}$ & 0 & 1 & 16 & 27 & 44 \\
\hline & $\%$ & $0,0 \%$ & $0,7 \%$ & $10,7 \%$ & $18,0 \%$ & $29,3 \%$ \\
\hline Alto & $\mathrm{n}$ & 0 & 1 & 22 & 18 & 41 \\
\hline & $\%$ & $0,0 \%$ & $0,7 \%$ & $14,7 \%$ & $12,0 \%$ & $27,3 \%$ \\
\hline Total & $\mathrm{n}$ & 7 & 7 & 70 & 66 & 150 \\
\hline & $\%$ & $4,7 \%$ & $4,7 \%$ & $46,7 \%$ & $44,0 \%$ & $100,0 \%$ \\
\hline
\end{tabular}

Rho $=.281 ; \mathrm{p}=.000$

En la tabla 5, se observa que del 100\% de la población, el 21,3\% de los adultos mayores tiene un nivel bajo en la dimensión apoyo emocional y son dependientes moderados con respecto a la dependencia funcional; por otro lado, el 0,07\% de adultos mayores tiene nivel alto y son dependientes severos. Con respecto a la prueba estadística r de Pearson señala un coeficiente de correlación de 0,281 y un p valor de 0,000 , siendo este menor a 0,05 , lo que permite aceptar la $\mathrm{H} 1$ y rechazar $\mathrm{H} 0$, quedando demostrando que existe relación entre la dimensión apoyo emocional y la dependencia funcional en adultos mayores de seis iglesias de IASD.

\section{Tabla 6}

Relación entre la dimensión apoyo instrumental y dependencia funcional en adultos mayores de seis iglesias adventistas del séptimo día, Lurigancho Chosica, 2019

\begin{tabular}{|c|c|c|c|c|c|c|}
\hline & & DT & DS & $\mathrm{DM}$ & & Total \\
\hline$\overline{\text { Apoyo }}$ & $\mathrm{n}$ & $64,0 \%$ & $64,0 \%$ & 34 & 24 & 70 \\
\hline Instrumental & $\%$ & & & $22,7 \%$ & $16,0 \%$ & $46,7 \%$ \\
\hline \multirow[t]{2}{*}{ Medio } & $\mathrm{n}$ & 1 & 1 & 25 & 35 & 62 \\
\hline & $\%$ & $0,7 \%$ & $0,7 \%$ & $16,7 \%$ & $23,3 \%$ & $41,3 \%$ \\
\hline \multirow[t]{2}{*}{ Alto } & $\mathrm{n}$ & 0 & 0 & 11 & 7 & 18 \\
\hline & $\%$ & $0,0 \%$ & $0,0 \%$ & $7,3 \%$ & $4,7 \%$ & $12,0 \%$ \\
\hline \multirow[t]{2}{*}{ Total } & $\mathrm{n}$ & $\mathrm{n}$ & 7 & 70 & 66 & 150 \\
\hline & $\%$ & $\%$ & $4,7 \%$ & $46,7 \%$ & $44,0 \%$ & $100,0 \%$ \\
\hline
\end{tabular}


En la tabla 6, se muestra que del 100\% de la población, el 23,3\% de los adultos mayores tiene un nivel de apoyo instrumental medio y son funcionalmente independientes; por otro lado, el 0,7\% de adultos mayores presentan un nivel medio de apoyo instrumental y tienen entre dependencia total y severa. Con respecto a la prueba estadística $r$ de Pearson, señala un coeficiente de correlación de 0,242 y un p valor de 0,003 , siendo este menor a 0,05 , lo que permite aceptar $\mathrm{la}_{1}$ y rechazar $\mathrm{H}_{0}$, quedando demostrando que existe relación entre la dimensión apoyo instrumental y la dependencia funcional en adultos mayores de seis iglesias adventistas del séptimo día.

Tabla 7

Relación entre la dimensión interacción social positiva y dependencia funcional en adultos mayores de seis iglesias adventistas del séptimo día, Lurigancho-Chosica, 2019

\begin{tabular}{|c|c|c|c|c|c|c|c|}
\hline \multirow{6}{*}{$\begin{array}{l}\text { Interacción } \\
\text { social } \\
\text { positiva }\end{array}$} & \multirow{6}{*}{$\begin{array}{l}\text { Bajo } \\
\text { Medio }\end{array}$} & & \multicolumn{4}{|c|}{ Dependencia funcional } & \multirow[b]{2}{*}{ Total } \\
\hline & & & \multirow{2}{*}{$\frac{\mathrm{DT}}{7}-$} & \multirow{2}{*}{$\frac{\text { DS }}{3}$} & \multirow{2}{*}{$\frac{\mathrm{DM}}{22}$} & \multirow{2}{*}{ I } & \\
\hline & & $\mathrm{n}$ & & & & & 44 \\
\hline & & $\% \mathrm{n}$ & $4,7 \%$ & $2,0 \% 3$ & $14,7 \%$ & $8,0 \%$ & $29,3 \%$ \\
\hline & & & 0 & & 23 & 27 & 53 \\
\hline & & $\%$ & $0,0 \%$ & $2,0 \%$ & $15,3 \%$ & $18,0 \%$ & $35,3 \%$ \\
\hline & Alto & $\mathrm{n}$ & 0 & 1 & 25 & 27 & 53 \\
\hline & & $\%$ & $0,0 \%$ & $0,7 \%$ & $16,7 \%$ & $18,0 \%$ & $35,3 \%$ \\
\hline Total & & $\mathrm{n}$ & 7 & 7 & 70 & 66 & 150 \\
\hline & & $\%$ & $4,7 \%$ & $4,7 \%$ & $46,7 \%$ & $44,0 \%$ & $100,0 \%$ \\
\hline
\end{tabular}

rho $=.220 ; \mathrm{p}=.007$

En la tabla 7, se muestra que del 100\% de la población, el 18,0\% de los adultos mayores tiene un nivel alto en la dimensión interacción positiva y son funcionalmente independientes, y un grupo minoritario de $0,7 \%$ con dependencia severa .Con respecto a la prueba estadística $r$ de Pearson, señala un coeficiente de correlación de 0,220 y un p valor de 0,007 , siendo este menor a 0,05 , lo que permite aceptar la $\mathrm{H}_{1}$ y rechazar $\mathrm{H}_{0}$, demostrando que existe relación entre la dimensión interacción positiva y la dependencia funcional en adultos mayores de seis iglesias adventistas del séptimo día. 


\section{Discusión}

El apoyo social consiste en abordar necesidades tangibles, como la asistencia con el transporte, el hogar y el cuidado personal, así como el apoyo emocional, como ser escuchado, comprendido y consolado. Por lo tanto, el apoyo social, ha sido reconocido como un importante determinante social de la salud porque ayuda a las personas a alcanzar sus necesidades físicas y emocionales, y reduce los efectos de los eventos estresantes en su calidad de vida. Recientemente, muchos estudios han demostrado una relación entre el apoyo social y la salud (mortalidad, las enfermedades crónicas, la cognición, los síntomas depresivos y el bienestar). De esta manera, las intervenciones dirigidas al apoyo social pueden ser una prioridad para mejorar el bienestar de las personas mayores y maximizar su salud y capacidad funcional.(Dai et al., 2016).

Los resultados del estudio revelaron que, el 38,7\% de los adultos mayores de las iglesias adventistas del Inti presentaron un apoyo social bajo, mientras que un 30,7\% de medio a alto. Resultados similares se encontraron Llange (2017) y Villalobos (2014) en investigaciones hechas en Perú. Según Figueroa (2018), estos resultados indican que los adultos mayores, en su mayoría, no reciben expresiones de afecto, cariño y seguridad de su red de apoyo. En torno a la dependencia funcional, los resultados evidenciaron que el 46,7\% de los adultos mayores lo calificaron como moderado, mientras que el 44\% fue independiente. De igual manera, Jirón y Palomares (2018) en Lima, reportaron que el $41,1 \%$ de los adultos mayores manifestaron dependencia de moderada a severa.

Desde otro orden de cosas, Doubova, Espinosa y Flores (2010), los principales factores de riesgo para la dependencia funcional son la edad avanzada, el sexo femenino, la baja alfabetización, la carga de la enfermedad, la depresión, la visión y el deterioro cognitivo, la limitación funcional de las extremidades inferiores, la mala salud auto percibida, el bajo nivel físico actividad, tabaquismo y baja frecuencia de contactos sociales. Si bien es cierto, la dependencia se evalúa y clasifica de diferentes maneras, en términos generales, cuanto mayor es la dependencia, mayores son las consecuencias negativas para el adulto mayor, su familia y la sociedad.

Cabe resaltar, que la mayor parte del cuidado de los adultos mayores funcionalmente dependientes depende de sus familiares y amigos, y pueden o no recibir un apoyo institucional, que proviene de los beneficios de la seguridad social o los programas sociales. Tal apoyo no es universal y no necesariamente cumple con sus necesidades sociales y de salud (Bernardes, Matos \& Goubert, 2017).

En cuanto a la relación entre las variables apoyo social y dependencia funcional, se encontró que existe una relación significativa con un p valor $=0.000$, donde se observó que el apoyo social es bajo en los adultos mayores con dependencia moderada, lo cual se contrapone con los datos 
sociodemográficos obtenidos, donde en su mayoría los adultos mayores son adventistas, casados , viven con sus esposo/a y/o hijos; por lo cual, se espera que en relación a sus creencias y valores sobre una imagen idealizada de la vejez y de la familia el apoyo social sea bueno . De igual manera, Arroyo y Ribeiro (2011) confirman que en los adultos mayores con dependencia física y necesidades de cuidado, la familia es la principal fuente de apoyo. Sin embargo, los cambios sociales, económicos y políticos a los que se han enfrentado las familias en las últimas décadas, han puesto en riesgo la capacidad de ésta para otorgar apoyo suficiente a sus familiares mayores.

En cuanto al análisis de las dimensiones del apoyo social, se encontró relación entre el apoyo emocional ( $\mathrm{p}=0,000)$, instrumental $(\mathrm{p}=0,003)$, interacción social positiva ( $\mathrm{p}$ valor $=0,007)$ y la dependencia funcional. Esto evidencia que una persona dependiente funcional recibe o necesita un tipo de apoyo social, ya sea emocional, afectiva, instrumental o de interacción. Lo que permite dar inclusión a este grupo etario para mantener en desarrollo las capacidades físicas y mentales de este. Así también, Ravello (2018) halló resultados similares y Pereira y Lost (2012) diferentes.

Por ello, para Muramatsu, Yin y Hedeker (2010), la disminución de la función física y cognitiva representa una gran amenaza para la vida independiente de los adultos mayores y sus cónyuges. En particular, los declives funcionales rápidos pueden invocar incertidumbres y, posiblemente, desesperanza sobre la vida independiente, con poco tiempo para adaptarse a la situación. Tener a alguien en quien confiar cuando lo necesite es probable que reduzca los efectos del estrés asociados con la disminución de la función.

\section{Conclusiones}

En relación con los objetivos, se obtuvo las siguientes conclusiones: el nivel de apoyo social fue bajo en adultos mayores de seis iglesias adventistas del séptimo día. El grado de dependencia funcional fue moderada en adultos mayores de seis iglesias adventistas del séptimo día. Asimismo, existe relación significativa, entre apoyo social y dependencia funcional, en adultos mayores de seis iglesias adventistas del séptimo día.

Por otro lado, existe relación significativa, entre la dimensión apoyo emocional y dependencia funcional, en adultos mayores de seis iglesias adventistas del séptimo día. De manera similar, existe relación significativa, entre la dimensión apoyo instrumental y dependencia funcional, en adultos mayores de seis iglesias adventistas del séptimo día. Además, existe relación significativa, entre la dimensión interacción social positiva y dependencia funcional, en adultos mayores de seis iglesias adventistas del séptimo día. Finalmente, existe relación significativa, entre la dimensión apoyo efectivo y dependencia funcional, en adultos mayores de seis iglesias adventistas del séptimo día. 


\section{Referencias}

Alvarado García, A. M., \& Salazar Maya, Á. M. (2014). Análisis del concepto de envejecimiento. Gerokomos, 25 (2), 57-62. https://doi.org/10.4321/s1134-928x2014000200002

Arroyo, M. \& Ribeiro, M. (2011). Apoyo familiar en adultos mayores con dependencias. Ciencia UANAL, 14 (3), 297-305.

Asamblea Médica Mundial (AMM). (2014). Declaración de Helsinki de la AMM - principios éticos para las investigaciones médicas en seres humanos. El Diario Del Colegio Americano de Dentistas, 81 (3), 14. https://www.wma.net/es/policies-post/declaracion-de-helsinki-de-laamm-principios-eticos-para-las-investigaciones-medicas-en-seres-humanos/

Baca, D. (2016). Confiabilidad y validez del cuestionario de apoyo social en pacientes con cáncer de Trujillo. Revista de Investigación en Psicología, 19 (1), 177. https://doi.org/10.15381/rinvp.v19i1.12452

Bernardes, S., Matos, M., \& Goubert, L. (2017). Older adults' preferences for formal social support of autonomy and dependence in pain: development and validation of a scale. European Journal of Ageing, 14 (3), 257-268. https://doi.org/10.1007/s10433-017-0411-X

Cerquera, A., \& Uribe, A. (2017). Apoyo social percibido y dependencia funcional en el adulto mayor con dolor. Diversitas: Perspectivas en Psicología, 13 (2), 215-227. https://doi.org/10.15332/s1794-9998.2017.0002.06

Dai, Y., Zhang, C., Zhang, B., Li, Z., Jiang, C., \& Huang, H. (2016). Social support and the self-rated health of older people A comparative study in Tainan Taiwan and Fuzhou Fujian province. Medicine (United States), 95 (24). https://doi.org/10.1097/MD.0000000000003881

Díaz, M. (2015). Habilidad de cuidado y carga del cuidador primario informal familiar de los pacientes con enfermedad crónica del "Programa Familiar Acompañante" del hospital Nivel II Vitarte - EsSalud, 2015 [Tesis de maestría, Universidad Peruana Unión]. http://repositorio.upeu.edu.pe/handle/UPEU/843

Doubova, S., Espinosa, P., \& Flores, S. (2010). Social network types and functional dependency in older adults in Mexico. BMC Public Health, 10, 104-112. https://doi.org/10.1186/14712458-10-104

Figueroa, G. (2018). Apoyo social en los adultos mayores institucionalizados del Centro de Atencion Residencial Geronto Geriatrico Ignacia Rodulfo Viuda de Canevaro del Distrito del Rimac, Periodo 2018 [Tesis de licenciatura, Universidad Inca Garcilaso de la Vega]. http://repositorio.uigv.edu.pe/handle/20.500.11818/3314

Flores, M. E., Decenas, K., Avalos, M. L., Colunga, C., Vega, M. G., \& Meza, I. J. (2016). Apoyo social percibido y envejecimiento activo en hombres y mujeres de una comunidad rural. Revista 
Electrónica de Psicología Iztacala, $19 \quad$ (4), 1316-1332.

http://www.revistas.unam.mx/index.php/repi/article/view/57952/51299

Gallardo, L. (2013). Apoyo social y etnicidad en personas mayores de la región de Arica y Parinacota-Chile: una propuesta para la intervención en trabajo social [Tesis doctoral, Universidad Complutense de Madrid]. https://eprints.ucm.es/id/eprint/20100/1/T34359.pdf García, M., Campos, J., De Dios, J., Escobar, M., Fernández, A., Gómez, C., Mainar, R., Merino, M., Molinero, S., Moya, A., Ramos, J., Ramos, P., Sanz, E., \& Valle, M. (2011). Guía Práctica de la Ley de Dependencia. Ley de promoción de la autonimia personal y atención a las personas en situación de dependencia. Madrid: Sociedad Española de Geriatría y Gerontología.

González, F., Masaad, C. \& Lavanderos, F. (2009). Estudio nacional de la Dependencia en las Personas Mayores. Chile. http://www.riicotec.org/InterPresent1/groups/imserso/documents/binario/estudionacionald eladependencia.pdf

Hernández, R; Fernández, C, Baptista, M, \& Méndez, S. (2014). Metodología de la Investigación Mexico D.F.: McGraw-Hill.

Instituto Nacional de Estadística e Informática. (2018). Adultos mayores de 70 y más años de edad, que viven solos sobre la base de los resultados de los Censos Nacionales 2017: XII de Población, VII de Vivienda y III de Comunidades Indígenas. Perú: 2018. Lima: INEI. https://www.inei.gob.pe/media/MenuRecursivo/publicaciones_digitales/Est/Lib1577/Libro01 .pdf

Jiménez, B., Eugenia, L., Ávalos, F. \& Campos, L. (2016). Dependencia funcional y percepción de apoyo familiar en el adulto mayor. Atención Familiar, 23 (4), 129-133. https://doi.org/10.1016/j.af.2016.08.002

Jirón, \& Palomares. (2018). Estilos de vida y grado de dependencia en el adulto mayor en el centro de atención residencial geronto-geriátrico Ignacia Rodulfo viuda de Canevaro, Rimac 2018 [Tesis de licenciatura, Universidad Norbert Wiener]. http://repositorio.uwiener.edu.pe/bitstream/handle/123456789/2416/TESIS\%20Jir\%c3\%b3 n\%20Jackeline\%20-\%20Palomares\%20Gisela.pdf?sequence=1\&isAllowed=y

Kamitsuru, S., \& Herdman, H. (2018). Diagnosticos de enfermagem da Nanda - I. Brasil: Artmed Editora Ltda. http://www.faesb.edu.br/biblioteca/wp-content/uploads/2020/03/NANDA-I2018_2020.pdf

Knight, G. (2002). Filosofía y Educación. Una introducción a la perspectiva cristiana. Miami, EE.UU.: Asociación Publicadora Interamericana. 
Lema, H. F. (2018). Dependencia funcional y depresión en adultos mayores del servicio de geriatría del Hospital III Regional Honorio Delgado, Arequipa, Perú 2018 [Tesis de licenciatura, Universidad Católica de Santa María]. http://tesis.ucsm.edu.pe/repositorio/bitstream/handle/UCSM/7517/70.2330.M.pdf?sequenc $\mathrm{e}=1 \&$ isAllowed $=\mathrm{y}$

Llange, J. (2017). Apoyo social y el grado de depresión en pacientes adultos de las salas de hospitalización del Hospital Vitarte durante el periodo junio - noviembre del 2016 [Tesis de licenciatura, Universidad Ricardo Palma]. https://www.scribd.com/document/391447050/APOYO-SOCIAL-Y-EL-GRADO-DEDEPRESION-EN-PACIENTES-ADULTOS-pdf

Marín, D. (2016). Bienestar psicológico y apoyo social percibido del cuidador familiar de adultos mayores [Tesis de maestría. Universidad de Concepción]. http://repositorio.udec.cl/jspui/handle/11594/2181

Meza, G. (2016). Capacidad funcional para desarrollar actividades de la vida diaria, según edad y sexo en adultos mayores que acuden a un centro de atención al adulto mayor. Villa María del Triunfo - 2016 [Tesis de licenciatura. Universidad Nacional Mayor de San Marcos]. https://cybertesis.unmsm.edu.pe/bitstream/handle/20.500.12672/5892/Meza_lg.pdf?sequen ce $=1 \&$ isAllowed $=\mathrm{y}$

Ministerio de Salud. (2014). Adultos mayores activos y saludables. http://www.minsa.gob.pe/portada/Especiales/2014/adulto/_cont.asp?pg=1

Ministerio de Salud, \& Dirección General de Epidemiología. (2013). Análisis de situación de salud del Perú. Biblioteca Nacional del Perú. https://doi.org/10.1590/S0036-36342011000500003

Muramatsu, N., Yin, H., \& Hedeker, D. (2010). Functional declines, social support, and mental health in the elderly: Does living in a state supportive of home and community-based services make a difference? Social Science and Medicine, 70 (7), 1050-1058. https://doi.org/10.1016/j.socscimed.2009.12.005

Naranjo, C. Y., Concepción, J. A. \& Rodríguez, M. (2017). La teoría Déficit de autocuidado: Dorothea Elizabeth Orem. Gaceta Médica Espirituana, $19 \quad$ (3), 89-100. http://scielo.sld.cu/pdf/gme/v19n3/GME09317.pdf

Organización Mundial de la Salud. (2020). Envejecimiento y ciclo de vida. https://www.who.int/ageing/about/facts/es/

Pereira, T. R. \& Iost, S. C. (2012). The relationship between social support and functional capacity in elderly persons with cognitive alterations. Revista Latino-Americana de Enfermagem, 20 (4), 677-684. http://www.scielo.br/pdf/rlae/v20n4/es_07.pdf 
Ravello, I. (2018). Apoyo social y satisfacción con la vida en adultos mayores del distrito de San Juan de Miraflores, Acta Psicológica Peruana, 2 (2), 217 -234. http://revistas.autonoma.edu.pe/index.php/ACPP/article/view/76/58

Rojas, J. (2017). Cuidados e intervenciones de enfermería enfocados en las necesidades de salud del adultos mayor, bajo la Teoría de Kristen M. Swanson [Tesis doctoral, Universidad Autónoma del Estado de México]. http://ri.uaemex.mx/bitstream/handle/20.500.11799/99139/TESIS\%20DOCTORADO\%20 JESSICA\%20ROJAS.\%2031\%20MAYO\%202017.pdf?sequence=1\&isAllowed=y

Toapanta, K. B. (2015). Nivel de dependencia funcional del adulto mayor con trastorno mental utilizando la escala de Barthel en usuarios de las residencias asistidas del instituto de neurociencias año 2015 [Tesis de licenciatura, Universidad de Guayaquil]. http://repositorio.ug.edu.ec/bitstream/redug/11000/1/CD\%20334\%20TOPANTA\%20MENDOZA\%2c\%20KARINA\%20BEATRIZ.pdf

Trigás, M., Ferreira, L. \& Meijide, H. (2011). Escalas de valoración funcional en el anciano. Galicia Clinica/Sociedad Galega de Medicina Interna, 11 (1), 11-16. https://galiciaclinica.info/pdf/11/225.pdf

Unsar, S., Dindar, I. \& Kurt, S. (2015). Activities of daily living, quality of life, social support and depression levels of elderly individuals in Turkish society. Journal of the Pakistan Medical Association, 65 (6), 642-646. https://jpma.org.pk/PdfDownload/7380

Vargas, L. \& Vargas, C. (2017). Apoyo social y bienestar psicológico en adultos mayores que asisten a los CIAM de Lima metropolitana. Universidad Peruana de Ciencias Aplicadas $(U P C)$ [Tesis de licenciatura, Universidad Peruana de Ciencias Aplicadas]. https://repositorioacademico.upc.edu.pe/bitstream/handle/10757/622168/VARGAS_AC.p $\mathrm{df}$ ? sequence $=5 \&$ isAllowed $=\mathrm{y}$

Velazco, L. \& Carrera, T. (2017). Dependencia fisca y deterioro cognitivo en los adultos mayores de la casa hogar "misioneros de amor", Chaclacayo, 2016 [Tesis de licenciatura, Universidad Peruana Unión]. http://repositorio.upeu.edu.pe/handle/UPEU/415

Villalobos, E. V. (2014). Apoyo social en los adultos mayores de un centro de salud de José Leonardo Ortiz-Chiclayo, 2014 [Tesis de licenciatura, Universidad Católica Santo Toribio de Mogrovejo].

White, E. G. (2011). El ministerio de curación. Buenos Aires, Argentina: ACES.

Zapata, B., Delgado, N., \& Cardona, D. (2016). Apoyo social y familiar del adulto mayor del área urbana. Angelópolis, Antioquia 2011. Revista de Salud Pública, 17 (6), 848-860. https://doi.org/10.15446/rsap.v17n6.34739 